\title{
A proposed management algorithm for late-onset efavirenz neurotoxicity
}

\author{
H M Cross, MB ChB, MSc, Dip HIV Man (SA), FC Neurol (SA); S Chetty, MB ChB; M T Asukile, BSc, MB ChB; H S Hussey, MB ChB; \\ E B Lee Pan, MB ChB, MMed (Neurol); L M Tucker, MB ChB, MSc, FCP (SA), PhD
}

Division of Neurology, Groote Schuur Hospital and Faculty of Health Sciences, University of Cape Town, South Africa

Corresponding author: L M Tucker (lmaskewtucker@gmail.com)

\begin{abstract}
A high proportion of HIV-positive patients in South Africa receive concomitant efavirenz (EFV) and isoniazid (INH) therapy. EFV is metabolised in the liver via CYP2B6, and genetic polymorphism of CYP2B6 is known to result in slowed metabolism of the drug. INH is also metabolised in the liver, causing inhibition of a pathway that plays an important role in slow EFV metabolisers. Concomitant INH use therefore affects plasma levels of EFV. EFV is well known to cause neuropsychiatric side-effects on initiation, and a recent adult case series described late-onset neurotoxicity in the form of subacute ataxia and encephalopathy in patients treated with EFV for a median of 2 years, in association with toxic plasma levels of the drug. We have seen an increase in cases of EFV toxicity presenting to our neurology referral unit. All cases have been in the context of recent initiation of concomitant INH. We therefore conducted a retrospective case record audit to describe these seven cases with the additional advantage of tertiary-level assessment. We outline the clinical features and investigation results, as well as outcomes after EFV was stopped. Our main objectives are to highlight the probable role of concomitant INH use in the development of this syndrome, and to suggest that only limited work-up may be warranted in suspected cases.
\end{abstract}

S Afr Med J 2018;108(4):271-274. DOI:10.7196/SAMJ.2018.v108i4.12914

Since September 2016, all individuals in South Africa (SA) who are diagnosed as HIV-positive are immediately eligible for antiretroviral therapy (ART), regardless of CD4+ count. The current preferred first-line regimen is a fixed-dose combination tablet comprising three antiretroviral agents, including efavirenz (EFV) ${ }^{[1]}$ Isoniazid (INH) is being increasingly used as tuberculosis (TB) prophylaxis, especially in HIV-positive individuals. This means that a high proportion of patients in SA are likely to be receiving concomitant EFV and INH therapy. We need to be aware of potential medication-related toxicity syndromes, especially in the context of polypharmacy.

$\mathrm{EFV}$ is a non-nucleoside reverse transcriptase inhibitor that is metabolised in the liver via the cytochrome p450 system, predominantly via CYP2B6. ${ }^{[2,3]}$ It has a long half-life and is therefore prescribed as a once-daily dose of $600 \mathrm{mg}$. However, genetic differences in the pharmacokinetics of EFV are increasingly being recognised. ${ }^{[2]}$

EFV is well known to cause neuropsychiatric side-effects. These typically occur (in $\sim 40 \%$ of cases) on initiation of the drug, but are usually mild and self-limiting within the first few weeks of exposure. ${ }^{[4]}$

A paediatric case series has described late-onset neurotoxicity associated with toxic EFV levels in the form of subacute ataxia in children treated with EFV-containing antiretroviral regimens for more than a year. ${ }^{[5]}$ A more recent case series from Klerksdorp, SA, ${ }^{[6]}$ described late-onset ataxia and encephalopathy in adult patients treated with EFV for a median of 2 years, in association with toxic plasma levels of the drug. These patients were all female with low body weight. Possible reasons proposed for susceptibility to EFV neurotoxicity included low body weight and polymorphism of the liver enzyme CYP2B6, which is known to result in slowed metabolism of the drug. ${ }^{[7]}$ Two common polymorphisms in CYP2B6 (516G $>\mathrm{T}$ and T983C) slow EFV metabolism and have been shown to occur with increased frequency in people with black African genetic ancestry. ${ }^{[3,8]}$ These polymorphisms probably also play a role in the acute toxicity, and a case of new-onset psychosis following recent initiation of EFV has been described in the context of confirmed CYP2B6 516G $>$ T polymorphism. ${ }^{[9]}$

INH is also metabolised in the liver via the cytochrome p450 system. It inhibits the CYP2A6 enzyme. Although a small proportion of EFV is normally metabolised via this enzyme, this pathway may have a more important role in slow metabolisers with polymorphisms affecting CYP2B6. Concomitant INH use therefore affects plasma levels of EFV, especially in slow metabolisers. This has previously been noted in a pharmacodynamic study. ${ }^{[10,11]} \mathrm{INH}$ has also been independently associated with neuropsychiatric effects, occurring a median of 21 days after initiation of treatment. ${ }^{[12,13]}$

We have noted a recent increase in cases of EFV toxicity presenting to our tertiary neurology referral unit in the Western Cape Province, SA, over a relatively short period of time. All the cases occurred in the context of recent initiation of concomitant INH treatment. We therefore conducted a retrospective case record audit to describe these cases of late-onset EFV toxicity. We outline the clinical details (history and examination) and the results of investigations (laboratory results, electroencephalogram (EEG) findings, imaging and neuropsychological assessments), as well as outcomes after EFV was stopped. Comprehensive investigation is often undertaken in HIV-positive patients with impaired cognition and cerebellopathy, because the differential diagnosis is wide and some of these conditions could deteriorate significantly during the period in which EFV is withdrawn. Our main objectives are to highlight the probable role of concomitant use of INH in the development of this syndrome, and to suggest that where a diagnosis of EFV toxicity is strongly suspected, only limited work-up may be warranted.

Cases are defined as individuals presenting to the Groote Schuur Hospital neurology services with subacute neurological signs and 
symptoms, confirmed toxic levels of EFV, and no other cause found for their presentation. The study was approved by the University of Cape Town Human Research Ethics Committee (ref. no. 603/2017).

\section{Results}

A typical case is that of a 37-year-old woman, originally from Lesotho, known to be HIV-positive (CD4+ count $>500$ cells $/ \mu \mathrm{L}$, viral load $<20$ copies $/ \mathrm{mL}$ ) who had been on ART (tenofovir/emtricitabine/ efavirenz) for 5 years. Concomitant INH prophylaxis was initiated 1 month prior to symptom onset. She was admitted after developing subacute, progressive confusion and an unsteady gait over the course of 1 month.

On examination she appeared systemically well, but neurological examination confirmed that she was disorientated with psychomotor slowing, poor attention and blunted affect. Primitive reflexes were not elicited. The findings on cranial nerve examination were normal. Motor examination revealed normal tone and reflexes and full power. Sensation was normal. Co-ordination was abnormal, and she had horizontal- and upward-gaze nystagmus with left-sided dysmetria, bilateral intention tremor and abnormal heel-shin tests. Marked truncal ataxia with a broad-based ataxic gait was also noted.

On investigation, basic blood work-up including a CD4+/CD8+ ratio, thyroid-stimulating hormone (TSH) and vitamin $\mathrm{B}_{12}$ were normal. Syphilis serology (TPAb) was negative. Liver enzymes, a hepatitis screen, ammonia levels and an abdominal ultrasound scan were normal. Cerebrospinal fluid (CSF) analysis confirmed normal basic parameters, and a cryptococcal latex antigen test, tuberculosis GeneXpert assay and JC virus polymerase chain reaction were negative. Neither computed tomography (CT) nor magnetic resonance imaging (MRI) of the brain revealed significant abnormalities. Two EEGs revealed generalised, high-amplitude, rhythmical, moderate (predominantly theta) slowing consistent with encephalopathy. Plasma EFV levels were toxic $(>20 \mathrm{mg} / \mathrm{L}$; normal therapeutic range $1-4 \mathrm{mg} / \mathrm{L}$ ).

EFV and INH were immediately withdrawn (and EFV substituted with lopinavir/ritonavir (LPV/r). The patient also initially received highdose parenteral thiamine, with little effect. Initial clinical improvement was noted at the time of discharge (12 days after admission).

At follow-up 3 months later she showed marked improvement. She was living independently and seeking employment. Her affect was appropriate without psychomotor slowing. Montreal cognitive assessment screen was normal. Cerebellar examination was normal except for a subtle persisting intention tremor affecting her nondominant hand. A repeat EEG was normal.

A total of seven adult women with southern African ancestry presenting with subacute encephalopathy and cerebellar ataxia were identified between May 2017 and August 2017. All had been exposed to INH, either prophylactically or as part of TB treatment. Six of the seven women were HIV-positive with a suppressed viral load. One patient was HIV-negative but was prescribed both EFV-containing $\mathrm{ART}$ and INH at a community clinic following what appears to have been a false-positive HIV test.
The clinical picture was similar in all these cases, with marked truncal and gait ataxia and psychomotor slowing, resulting in severe functional impairment and inability to walk unaided. Four patients had nystagmus with abnormal smooth pursuit and saccadic overshoot. Two women exhibited additional mood and psychotic symptoms prior to the encephalopathic, ataxic presentation. One woman initially presented with psychosis and was admitted to the psychiatric ward before being referred for neurological assessment. The other woman initially presented with and was treated for depression as an outpatient (in hindsight this may have been a misdiagnosis of psychomotor slowing). Further characteristics of the included patients are summarised in Tables 1 and 2.

All the cases were investigated according to our neurology division practice. Blood tests included TSH, vitamin $\mathrm{B}_{12}$, TPAb, CD8+ count, liver function tests and ammonia levels. All the women had toxic plasma EFV levels $(>20 \mathrm{mg} / \mathrm{L})$. CSF was sampled in all cases and found to be normal (including JC virus/FTA-ABS/TB GeneXpert). $\mathrm{CT}$ and MRI brain imaging were performed in all cases. Two patients had non-contributory findings (one an old basal ganglia infarct and the other a left parietal FLAIR white-matter hyperintensity of undetermined significance on MRI).

EEG, performed in all cases, revealed distinct, moderately high amplitude, rhythmical, generalised (predominantly theta) slowing (except for one case in which the slowing was irregular). Formal neuropsychological assessments were performed in five cases. These yielded uniform findings of predominantly dysexecutive deficits and psychomotor slowing consistent with frontal lobe subcortical dysfunction.

All the women showed marked initial improvement in cerebellar and cognitive function a median of 14 days after EFV withdrawal.

\section{Discussion}

We have described a series of seven women on EFV-based ART with concomitant INH, who presented with a syndrome of subacute encephalopathy and cerebellopathy with toxic plasma EFV levels. The encephalopathy included disorientation and psychomotor retardation of varying severity, and psychosis in one case. Cerebellar dysfunction ranged from mild truncal ataxia to florid pan-cerebellar

\section{Table 2. Characteristics of the patients}

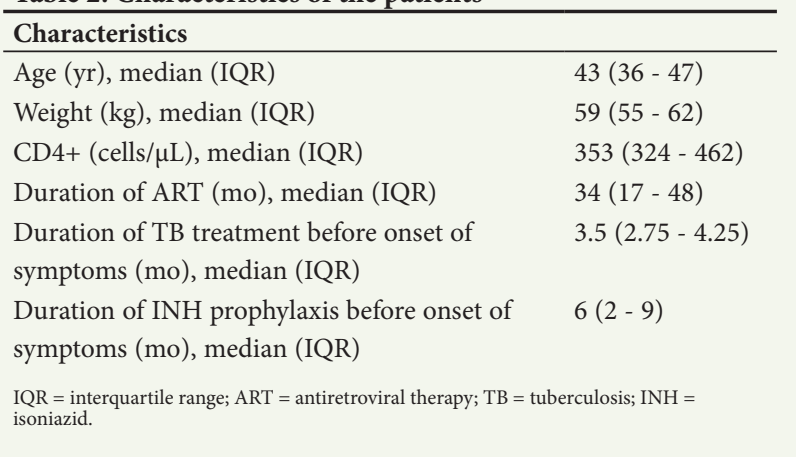

Table 1. Clinical characteristics of cases

\begin{tabular}{|c|c|c|c|c|c|c|c|}
\hline & Case 1 & Case 2 & Case 3 & Case 4 & Case 5 & Case 6 & Case 7 \\
\hline Cerebellar signs & +++ & ++ & +++ & + & +++ & + & ++ \\
\hline Neuropsychiatric signs/symptoms & +++ & +++ & +++ & ++ & +++ & + & +++ \\
\hline EEG generalised rhythmical slowing abnormality & ++ & ++ & ++ & ++ & ++ (irregular) & ++ & ++ \\
\hline Days to initial improvement (median 14) & 12 & 21 & 21 & 16 & 10 & 10 & 10 \\
\hline
\end{tabular}


dysfunction, including nystagmus. All the patients were virally suppressed with relatively well-preserved CD4+ counts (one patient was HIV-negative) and an otherwise negative diagnostic work-up. The patients all showed improvement within 14 days of EFV withdrawal, strengthening the hypothesis that drug toxicity was responsible.

Our patients were all women of southern African genetic ancestry with an age range of 30 - 55 years. Patients described previously in a similar case series were also all women ${ }^{[6]}$ It is unclear whether the proposed mechanism of EFV toxicity (resulting from slow metabolism of EFV due to polymorphisms of cytochrome CYP2B6) ${ }^{[2,7,14]}$ may have a sex predilection, as previous studies assessing this have yielded conflicting results. ${ }^{[2,7,14]}$ Some researchers suggest that non-Caucasian females are at high-risk for toxicity even after correcting for age and body weight. ${ }^{[14]}$ Factors such as differences in compliance and healthseeking behaviour between men and women may also play a role. There are almost twice as many women (compared with men) on $\mathrm{ART}$ in $\mathrm{SA},{ }^{[15]}$ and women have higher rates of adherence to ART. ${ }^{[15,16]}$

Our patients all had considerable (asymptomatic) exposure to ART prior to presentation (although this varied widely from 5 months to 4 years), qualifying our definition of 'late-onset' EFV toxicity. Our patients had all recently been commenced on INH (either in the form of prophylaxis or as part of TB treatment) with an average of 3 months' INH use prior to symptom onset. This implies a temporal relationship between INH commencement (in the context of EFV use) and onset of symptoms. While we did consider the possibility that INH toxicity might be the cause of the symptoms, we concluded that the EFV was more likely to be causative given the clinical improvement observed in two patients in whom INH was continued after withdrawal of EFV. Furthermore, limited studies of INHinduced cerebral toxicity document earlier onset of symptoms (mean 21 days). ${ }^{[11,13]} \mathrm{We}$ were unable to measure INH blood levels in our patients.

Unlike a similar case series of EFV toxicity, ${ }^{[6]}$ our patients had an average body weight of $55 \mathrm{~kg}$, with none weighing less than $40 \mathrm{~kg}$. They all received the recommended dose of EFV (600 mg/d). A lower dose of EFV (400 mg/d) has been shown to be non-inferior to $600 \mathrm{mg} / \mathrm{d}$ in terms of viral suppression, ${ }^{[17]}$ and it has been suggested that this lower dose may reduce the risk of toxicity in underweight individuals.

We are unable to comment on whether toxic EFV levels may be found in asymp- tomatic individuals, as EFV levels are not routinely measured as part of therapeutic drug monitoring. ${ }^{[18]}$

Based on our observations, we have proposed an algorithm (Fig. 1) for the clinical management of patients presenting with possible EFV-induced late-onset ataxia. It is important that systemic diseases first be excluded. Red flags of older age, low CD4+ count and pyrexia make EFV toxicity less likely, and an alternative diagnosis should be more actively sought. However, once systemic diseases have been excluded, and if both the CSF and CT brain scan are normal, we propose that it could be appropriate to wait for the results of the EFV levels and not investigate the patient further while the levels are pending. This would reduce the costs of investigation and unnecessary transfer of patients to higher-level care facilities. Once there is a suspicion of EFV toxicity, the drug should be discontinued immediately. Alternatives such as nevirapine, LPV/r or dolutegravir can be used, depending on the patient's viral load and clinical profile, including the presence of comorbid TB.

EEG is not required to make the diagnosis, but is useful in cases where it is clinically difficult to distinguish encephalopathy from psychiatric disorders.

This is a report of only a few cases observed at a single neurology referral centre, and the conclusions that can be drawn are therefore limited. We were not able to determine INH levels or EFV metaboliser genotype. Our observations do, however, corroborate those described in a similar group of patients. ${ }^{[6]}$ We would also like to highlight the likely role of INH in precipitating this syndrome, and in our experience low body weight is not a necessary factor. The findings from this report support consideration of the removal of EFV as first-line treatment for HIV in SA. In the interim, clinicians need to be aware of the potential for the development of this syndrome in patients on EFV, especially those concomitantly managed with INH.

Acknowledgements. We thank Prof. Gary Maartens and Dr Isma-eel Ebrahim of the Groote Schuur Hospital Division of Clinical Pharmacology for their advice and input.

Author contributions. HMC, SC, MTA, HSH and EBLP designed the study and reviewed all the drafts. HMC, SC, MTA and HSH compiled the patient data and wrote various sections of

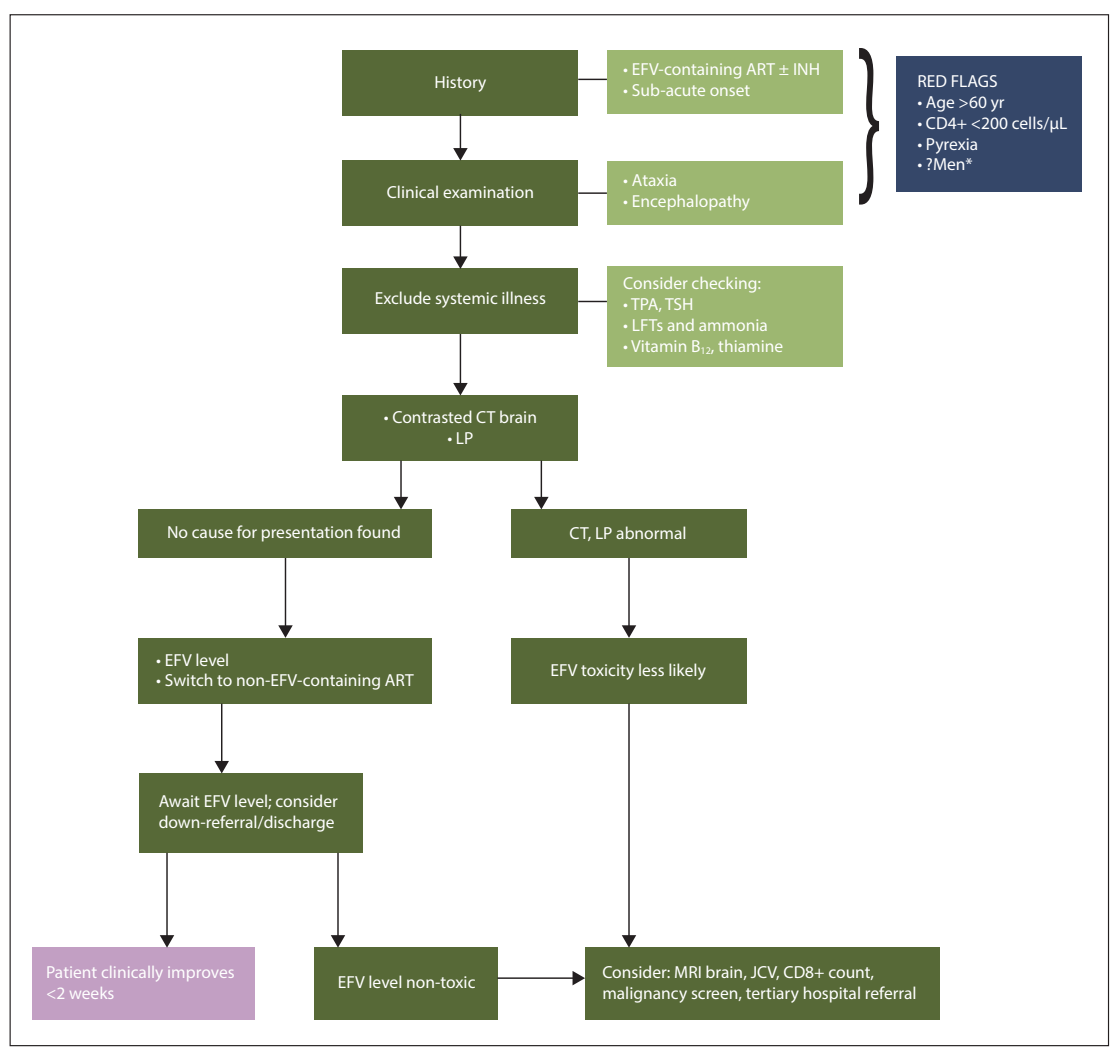

Fig. 1. Algorithm for the clinical management of suspected EFV neurotoxicity. $(E F V=$ efavirenz; $A R T=$ antiretroviral therapy; INH = isoniazid; TPA = treponemal antibodies; $T S H=$ thyroid-stimulating hormone; LFTs = liver function tests; $C T=$ computed tomography; $L P=$ lumbar puncture; $M R I=$ magnetic resonance imaging; JCV $=J C$ virus polymerase chain reaction. ${ }^{*}$ All previously described adult cases are women, although numbers are limited.) 
the paper. LMT assisted with final editing. All authors were involved in the management of the patients.

\section{Funding. None.}

\section{Conflicts of interest. None.}

1. Meintjes G, Moorhouse MA, Carmona S, et al. Adult antiretroviral therapy guidelines 2017. South Afr J HIV Med 2017;18(1):2078-6751. https://doi.org/10.4102/sajhivmed.v18i1.776

2. Arab-Alameddine M, di Iulio J, Buclin T, et al. Pharmacogenetics-based population pharmacokinetic analysis of efavirenz in HIV-1-infected individuals. Clin Pharmacol Ther 2009;85(5):485-494. https:// doi.org/10.1038/clpt.2008.271

3. Sinxadi PZ, Leger PD, McIlleron HM, et al. Pharmacogenetics of plasma efavirenz exposure in HIV infected adults and children in South Africa. Br J Clin Pharmacol 2015;80(1):146-156. https://doi. org/10.1111/bcp. 12590

4. Apostolova N, Funes HA, Blas-Garcia A, Galindo MJ, Alvarez A, Esplugues JV. Efavirenz and the CNS: What we already know and questions that need to be answered. J Antimicrob Chemother 2015;70(10):2693-2708. https://doi.org/10.1093/jac/dkv183

5. Hauptfleisch MPK, Moore DP, Rodda JL. Efavirenz as a cause of ataxia in children. S Afr Med J 2015;105(10):876-877. https://doi.org/10.7196/SAMInew.8780

6. Variava E, Sigauke FR, Norman J, et al. Brief Report: Late efavirenz-induced ataxia and encephalopathy: 6. Variava E, Sigauke FR, Norman J, et al. Brief Report: Late efavirenz-induced ataxia and encephalopathy:
A case series. J Acquir Immune Defic Syndr 2017;75(5):577-579. https://doi.org/10.1097/ A case series. J Ac

7. Gounden V, van Niekerk C, Snyman T, George JA. Presence of the CYP2B6 516G>T polymorphism, increased plasma efavirenz concentrations and early neuropsychiatric side effects in South Africa HIV-infected patients. AIDS Res Ther 2010;7(1):32-41. https://doi.org/10.1186/1742-6405-7-32

8. Haas DW, Ribaudo HJ, Kim RB, et al. Pharmacogenetics of efavirenz and central nervous system side effects: An Adult AIDS Clinical Trials Group study. AIDS 2004;18(18):2391-2400.

9. Hasse B, Günthard HF, Bleiber G, Krause M. Efavirenz intoxication due to slow hepatic metabolism. Clin Infect Dis 2005;40(3):e22-e23. https://doi.org/10.1086/42703
10. Mcllleron HM, Schomaker M, Ren Y, et al. Effects of rifampin-based antituberculosis therapy on plasma efavirenz concentrations in children vary by CYP2B6 genotype. AIDS 2013;27(12):1933-1940. plasma efavirenz concentrations in children vary
https://doi.org/10.1097/qad.0b013e328360dbb4

11. Court $\mathrm{MH}$, Almutairi FE, Greenblatt DJ, et al. Isoniazid mediates the CYP $2 \mathrm{~B}^{*} 6$ genotypedependent interaction between efavirenz and antituberculosis drug therapy through mechanismbased inactivation of CYP2A6. Antimicrob Agents Chemother 2014;58(7):4145-4152. https://doi. based inactivation of CYP 2 A6
org/10.1128/aac.02532-14

12. Bhattacharyya S, Darby RR, Raibagkar P, Gonzalez Castro LN, Berkowitz AL. Antibiotic-associated encephalopathy. Neurology 2016;86(10):963-971. https://doi.org/10.1212/wnl.0000000000002455

13. Peter P, John M. Isoniazid-induced cerebellitis: A disguised presentation. Singapore Med J 2014;55(1):e17-e19. https://doi.org/10.11622/smedj.2013188

14. Burger D, van der Heiden $\mathrm{I}$, la Porte $\mathrm{C}$, et al. Interpatient variability in the pharmacokinetics of the HIV non-nucleoside reverse transcriptase inhibitor efavirenz: The effect of gender, race, and CYP2B6 polymorphism. Br J Clin Pharmacol 2006;61(2):148-154. https://doi.org/10.1111/j.13652125.2005.02536.x

15. Shisana O, Rehle T, Simbayi L, et al. South African National HIV Prevalence, Incidence and Behaviour Survey, 2012. Cape Town: HSRC Press. 2014.

16. Heestermans T, Browne IL, Aitken SC, Vervoort SC, Klipstein-Grobusch K. Determinants of adherence to antiretroviral therapy among HIV-positive adults in sub-Saharan Africa: A systematic review. BMJ Glob Health 2016;1(4):e00125. https://doi.org/10.1136/bmigh-2016-000125

17. Puls R, Amin J, Losso M, et al. Efficacy of $400 \mathrm{mg}$ efavirenz versus standard $600 \mathrm{mg}$ dose in HIV-infected, antiretroviral-naive adults (ENCORE1): A randomised, double-blind, placebocontrolled, non-inferiority trial. Lancet 2014;383(9927):1474-1482. https://doi.org/10.1016/s01406736(13)62187-x

18. Nettles RE, Kieffer TL, Parsons T, et al. Marked intraindividual variability in antiretroviral concentrations may limit the utility of therapeutic drug monitoring. Clin Infect Dis 2006;42(8):11891196. https://doi.org/10.1086/501458

Accepted 14 November 2017 